Paper:

\title{
The Influential Factors to Lower Attitude Pupil's Learning Behavior toward Physical Education Class
}

\author{
Satoshi Otomo* and Tomoya Ogawa* \\ ${ }^{*}$ Gunma University, Faculty of Education \\ 4-2, Aramaki, Maebashi, Gunma, 371-8510 Japan \\ **Hosono Elementary School Japan \\ 365, Arai, Matsuida, Usui, Gunma, 379-0216 Japan \\ E-mail: otomo@edu.gunma-u.ac.jp \\ [Received January 10, 2003; accepted January 28, 2003]
}

\begin{abstract}
Factors influential on students not positive toward physical education practice. The goal of this study is to identify factors influential on the behavior of boys and girls not positive toward physical education practice. Three categories of factors were confirmed in having influences to learning behavior of these students. Four boys and four girls in total, each one from 3rd to 6th grade elementary school students took part in our studies. They disliked physical education practice. Seventy classes were observed. Journals, interviews to teachers, and discussion with teachers are added. In addition to these, to promote studies, analysis of these data clarified that three categories of factors, that is, (1) difficulty level of motor task (2) learner group and (3) teaching principles of teachers are influential factors. These three categories had strong relation with motor skill, while they did not work positively for students, whose tendencies toward physical education is relatively low, to improve their learning behavior, and also they did not work for student motor skill to had been improved.
\end{abstract}

Keywords: attitude toward physical education class, learning behavior, qualitative research on teaching in physical education

[International Journal of Sport and Health Science Vol.1 (1) 76-81]

\section{Introduction}

One of the main objectives of PE education in schools in Japan is to nurture tendencies toward physical activity and sports [ Ministry of Education, Science and Culture, Government of Japan (1999)].

However, "The rate of 'Sports-Hater' (Con-group) in Japan is assumed to be about 2-10\%[Sakumoto and Shinozaki (1979, p.69)]." In a survey of upper graders in elementary schools and students in junior high schools, it has been found that about 6-9\% males and $12-18 \%$ females disliked taking physical activity and about 5-11\% males and 14-23\% females disliked PE classes [Jinzenji et at. (1993, p.27)].

In order to improve the above situation, efforts have been made to identify the cause of such negative attitudes toward physical activity and sports [Kobayashi (1970); Sakumoto and Shinozaki (1979); Hatano and Nakamura (1981); Jinzenji et al. (1993)]. As a result, two main categories of causes were revealed, namely, causes concerning the student/child and others concerning PE classes.

Remarks that require special attention in our effort to improve PE classes are: "Those students who do not enjoy PE classes tend to put the blame on teachers
[Kobayashi (1970, p.291)]." and "Part of the reason seemed to be the amplification of the inferior complex of children caused by their experience in PE classes [Hatano and Nakamura (1981, p.184)]."

However, the above studies did not offer concrete information toward the improvement of PE classes, as they did not review the causes of dislike of physical activity and/or PE class in the context of the incidents actually happening in PE classes.

It has been indicated that how a child enjoys $\mathrm{PE}$ classes and sports is closely related [Nishi and Kikkawa (1980)], and it is easily assumable that a child's tendency to PE classes is affected by the evaluation of PE class made by the child. On the other hand, it has been indicated that the evaluation of PE class made by the child is related to learning behaviors [Takahashi et al. (1989)]. Thus, it is assumable that a child's tendency to sports, tendency to PE classes, the evaluation of PE class made by the child, and learning behaviors are all closely related.

Accordingly, we expect that by improving learning behavior, the tendency to PE classes will improve, which in turn will improve tendency to physical activity and sports. 
It is necessary for the improvement of learning behavior to review factors that affect learning behavior, based upon studies focusing on the actual state of PE classes. As there are children who do not enjoy PE classes in Japan, it is necessary to start the needed review by focusing our attention on the above children.

Thus, the purpose of this study is to identify the factors that affect learning behaviors of children who do not like PE classes.

\section{Methods}

\subsection{Participants}

Classes participated in our study were 4 classes consisting of 1 class each of 3rd to 6th graders in Y Elementary School in X Prefecture. The classes were held between early October and mid December 2000. Teacher A, who gave the class to 3rd and 5th graders, is the classroom teacher of the 3rd graders and the head of the physical education of Y Elementary School (44 year old female with 22 years of experience as a teacher). Teacher B, who gave the class to 4 th and 6 th graders, is the classroom teacher of the 6th graders ( 37 year old male with 14 years of experience as a teacher).

We chose 1 male and 1 female from respective grades, or 8 in total as children who are not showing favorable attitudes toward PE classes, hereinafter called "unwilling children", based upon: (1) evaluation of attitudes made by teachers (on a scale of 1 to 3); (2) evaluation of overall PE classes made by teachers (on a scale of 1 to 3); (3) evaluation of attitudes using a questionnaire [Takada et al. (1991)]; (4) evaluation of likes and dislikes of type of sports using a questionnaire; (5) observing $3 \mathrm{PE}$ classes after the start of observation activities. They are Boy A and Girl B in the 3rd grade, Boy C and Girl D in the 4th grade, Boy E and Girl $\mathrm{F}$ in the 5th grade, and Boy $\mathrm{G}$ and Girl $\mathrm{H}$ in the 6th grade.

We proceeded with our study based upon consent of the principal, assistant principal, Teacher A, Teacher $\mathrm{B}$, the classroom teachers of the 4th and the 5th graders, all other teachers of Y Elementary School, and all guardians of all children of Y Elementary school on the implementation of the study.

\subsection{Observed Class Activities}

During the observation activities, 18 classes were given to 3rd graders, consisting of 4 units of study, 19 classes to 4 th graders, consisting of 4 units, 15 classes to 5th graders, consisting of 3 units and 16 classes to 6th graders, consisting of 3 units, while the combined PE class was given 1 each to 3 rd and 4 th graders as well as 5th and 6th graders. In total 70 classes, consisting of 14 units. No intervention was made during the classes observed.

\subsection{Collection of Data}

The learning behaviors of "unwilling children" were written down in detail concerning the classes observed (hereinafter called "journal"). The journal was kept after each class in sentences referring to notes written down while observing class activities. Additional comments were added after watching a video showing teachers' behaviors during the class. These tasks were done by the second writer.

Teachers took formal interviews before the start and after the end of observation (semistructured interview). The details of the interview before the start of the observation were recorded by taking down notes, while those after the observation were recorded using a voice recorder. The classroom teachers of the 4th and the 5 th graders took formal interviews (semistructured interview) before the start of the observation and recorded by taking down notes. The interview of teachers after the end of the observation was organized into a protocol. Member checks were put on the records of the interviews. In order to avoid the possible change of teacher behaviors toward unwilling children, we did not let them know which of the children we chose.

Characteristic learning behaviors of unwilling children were written down as a protocol through the discussion with teachers while recording the process with a voice recorder.

The teachers were equipped with a wireless microphone, and their words, activities and behaviors during the class were e recorded in a video. The shooting was carried out from a certain place in a distance as inconspicuously as possible. Questionnaire surveys were carried out before the start of the observation of all children who participated in the class without notifying them of the purpose of the study.

\subsection{Procedures of Qualitative Data Analysis}

The qualitative data obtained was analyzed in the following manner:

1) (1) Learning behaviors of unwilling children, behaviors of teachers and the other children toward unwilling children, incidents that involved unwilling children, and the overall atmosphere of the class were put down in the journal; (2) conjectures or subjective evaluations concerning the possible factors affecting the learning behaviors of unwilling children; (3) incidents that caused the above conjectures or subjective evaluations; (4) scenes of the class; and (5) participants in the class were put in the journal. The first 
writer checked all journals to confirm that all the above contents of the journal, i.e., (1) through (5), were clearly categorized for every class hour, and when the categorization was found to be not satisfactory, revisions were made repeatedly until the categorization was complete.

2) Characteristic learning behaviors of unwilling children were extracted from the journal. Interviews of the teachers before the start of the observation were referred to during the process.

3) Extracted learning behaviors that have behavioral characteristics were made into groups. As a result, 10 groups were identified.

4) Teachers and writers discussed if it is reasonable to regard the above 10 groups as the characteristic learning behaviors of unwilling children. As a result, it was determined that 9 of the groups could be reasonably regarded as such, while one could not.

5) The above 9 groups showed learning behaviors of unwilling children or incidents that involved them. Accordingly, superior concepts that cover groups were searched and the 9 groups were classified into 3 categories.

6) Concerning the above 3 categories, negative cases were searched for in order to confirm the reorganization of categories and restrictions on situations in the journal and in the characteristic learning behaviors extracted from the journal. Some negative cases were identified in respective categories, which were positioned as concepts for the refining of the 3 categories. The above are the procedures taken in identifying the 3 categories. Note that more than 6 out of 8 unwilling children showed learning behaviors that belonged to respective categories.

Thus, the data analysis was carried out inductively, and categories were gradually refined [Bogdan and Biklen (1992)].

\section{Results and Discussion}

Three categories were identified, namely, (1) the level of difficulty of motor task, (2) learner group, and (3) teaching principles of the teacher, as factors that affect the learning behaviors of unwilling children.

\subsection{Level of Difficulty of Motor Task}

The learning behaviors of unwilling children were highly affected by the level of difficulty of motor task and sports learned in the class. Their learning behaviors were not positive when they found the motor task not interesting or difficult. In these situations, unwilling children (1) tried to decrease the learning opportunities being practiced or played; (2) voiced aloud their inability to perform the motor tasks; (3) chose easier motor tasks and would not challenge more difficult ones.
On the contrary, when they found motor tasks interesting, or the motor tasks were something they were good at, unwilling children positively involved themselves in the class, were attentive to what the teacher was explaining, and offered opinions in group discussion. The following are the more detailed description of actual incidents that took place in the class:

- It was during the 4 th hour of the mat exercises for 4th graders. Girl D went to the place where they were to practice forward shifting with legs astride and stood at the end of the queue. She said, "I can't," when it was her turn. During the warmingup session in the 9th hour, Girl D would not do headstand, insisting, "No way. I can't."

- This was during the 3rd hour of track and field (long jump) for 5 th graders. Children practiced in three different places in order to learn three different motor tasks in the jump: i.e., the running speed before the take off, the strength when hitting the board, and how to force the body through the air. Girl F chose and practiced the movement of the body in the air. Children were supposed to run to the vaulting box, kick hard on the top of the box, and throw the body forward to take a suitable posture in the air. She did the running, climbing up of the vaulting box and going down in a continuously slow manner. After a while, Teacher A told her to do each of the three motor tasks in turn, but she kept practicing the same motor task until the class was over.

- It was the 2nd hour of the mat exercise for 6th graders. Boy $\mathrm{G}$ went to the place where the forward shifting with legs astride was practiced. $\mathrm{He}$ said, "I will stand at the end of the line," and did just that. Then, Teacher B told the children to practice the diving forward shifting. Boy $\mathrm{G}$ was again at the end of the line. When the team started the practice separately, he left the mat and just watched other children practice the motor task. During the other hours of the class also, he kept leaving the line when his turn came and just watched other children.

On the other hand, we observed occasions when unwilling children were actively involved in the practice. Here are a couple of such occasions:

- The unit of fundamental physical activities for the 3 rd graders included unicycle and stilts. During the first hour, they practiced riding on a unicycle. Girl B swiftly went and returned from the jungle gym to the monkey bars, covering about 14 meters as well as rode backwards for 2 meters. Girl B addressed to Teacher A, saying, "Look, ma'am. I can ride backwards." She did the backward ride 3 times. In the 2nd hour, they practiced walking on stilts. Girl B fell from the stilts after a couple of steps. In the 3rd and 4th hours, Teacher A told the children to practice both unicycle and stilts in turn, but Girl B kept practicing positively and specifically the unicycle.

- Boy $\mathrm{G}$, who is a 6th grader, did not participate 
positively in the practice concerning most of the motor tasks. However, as for the practice of longdistance running, he was always close to the teacher, listening carefully what was being said.

Learning behaviors in this category were found in 6 out of the 8 unwilling children.

\subsection{Learner Group}

The learner group tried to deter unwilling children's independent learning behaviors by (1) telling them what to do; (2) making their lower performance known.

The unwilling children, in most of the cases, obeyed the learner group. They seldom offered opinions in team activities. Some of them stayed together with their usual pals or stayed all alone when they were required to choose one of the motor tasks. The followings are some of the examples observed:

- It was the 5th hour to learn games (Kick Baseball) for the 3rd graders. Boy A was standing behind Boy $I$ in the field. When the in-team player got to the first base, Boy I told Boy A to go near the home base. Boy A asked Boy I, "What am I supposed to do?" Boy I never answered to this question, but after that, Boy $\mathrm{A}$ was always near the home base whenever his team was in the field.

- During the 2nd hour of track and field (relay race) for 5 th graders, teams decided the order of the runners and the distance to be covered. In the discussion of the team to which Girl F belonged, team members said, concerning the part Girl F was to cover, "The part that includes a curve (because nobody can run fast)," and Girl F obeyed. Girl F and Girl $\mathrm{K}$ went together to the starting line. Girl $\mathrm{K}$ addressed to Girl F, saying, "Hey F, here's your starting line," making her start from the forward most of all the other runners belonging to the other teams, although the next runners were supposed to start from the same line, which made the distance covered by Girl F the shortest.

- During the 3rd hour of soft volleyball for 6th graders, teams were standing in a circle and practicing overhand pass. In Girl H's team they were taking turns to pass the ball. After a while, Teacher B suggested, saying, "Now why don't you try to pass 20 times in a row?" The children loudly counted the number of the pass. In this motor task, Girl H did not have any chance to touch the ball. And in the 8th hour, Teacher B grouped the children into 2 teams to play a match. Girl $\mathrm{H}$ was in the front right. In the middle of the game, Boy $\mathrm{L}$ who was in the back right told Girl $\mathrm{H}$ to change the positions, and the latter immediately obeyed.

There were times when unwilling children acted differently from the instruction made by the learner group. Here are some examples:

- When children were practicing track and field (relay race), Boy $\mathrm{E}$ did not have many chances of practicing passing of the baton. The other children did not talk to him. However, when they were practicing long-distance running, children talked to him and cheered for him.

- During the mat exercise practice, Girl F stayed mostly alone. But when she successfully completed a forward shifting with legs astride for the first time, other children talked to her.

On other occasions, other children addressed to and/or encouraged unwilling children when the motor task was something he/she had interest in.

Learning behaviors in this category were observed concerning all the 8 unwilling children.

\subsection{Teaching Principles of the Teachers}

The unwilling children, while engaged in motor task, received general and positive feedback, rather than specific and skill feedback from the teacher. Some of them frequently received scolding from the teacher during the teaching episode of instruction. The following are some of the examples:

- It was the 3rd hour of mat exercise practice for 4th graders. When Boy $\mathrm{C}$ did a forward shifting, his body skewed to the left. Teacher B said, "C, give it all you've got. Mind your left hand next time, C." Boy $\mathrm{C}$ tried the move again with little improvement. Then the teacher said to him, "It might take some time before you could stand at the end of the shifting."

- This happened in the 2 nd hour of mat exercise practice for 6th graders. When Girl $\mathrm{H}$ did a forward shifting with legs astride, she did not make the shifting fast enough and accordingly although she raised herself a few inches from the mat, she could not stand up successfully. Teacher B said to her, "Can you make it and stand up?" and left. After a while the teacher told the children to practice the "diving forward shifting." When Girl $\mathrm{H}$ did the shifting, it was not a diving but an ordinary forward shifting. Teacher B said to her, "Try a little bit harder, and you can make it."

When the teacher gave feedback, the unwilling child tried to carry out a little harder motor task, and succeeded. But the effort was never repeated. The following are such occasions:

- In the 5th hour of mat exercise for the 5th graders, Girl F could not stand up successfully after some practice of the forward shifting with legs astride. Teacher A suggested to her, saying, "Push hard with your hands." She tried backward shifting with legs astride and stood up all right. Teacher A praised her, saying, "So now you know how to do backward shifting with legs astride." Girl F smiled a little and said, "Yes, ma'am." However, after that although she tried, she never successfully stood up at the end of a forward and a backward shifting with legs astride. And she never gained the motor skill before the unit of study is over. 
- During the 2nd hour of mat exercise for 6th graders, Boy G could not stand up at the end of a forward shifting with legs astride. Teacher B said, "You can do it. Stand up, G. You can do it, as you're keeping your legs nice and straight." When Boy G tried it again in front of Teacher $\mathrm{B}$, he could do it all right. Teacher B and Boy G made eye contact with each other and the teacher nodded and said, "Yes." However, Boy G was never successful after that until the end of the unit of study. We did not see him practice positively.

Teachers made minor reprimands to unwilling children during the teaching episode of instruction. Here are some examples:

- In the 5th hour of mat exercise for the 5th graders, children were made to show their skill one by one at a signal from the teacher. Teacher $\mathrm{A}$ had not scolded children for being noisy until she said to Boy E, "You watch your friend and clap your hands, E." Right after this remark, Boy E started talking with the boy sitting right behind him.

Now, why do the two teachers seldom give specific and skill feedback to unwilling children, but scold them during the teaching episode of instruction?

In the discussion after the observation, the teachers explained as follows:

Concerning the feedback:

Teacher A said: I think I addressed to the children in order to motivate them. ...Maybe, as you say, instructions concerning skills are insufficient. But in a way I am motivating them all right.

Teacher B said: I am afraid it is just a lip effort when I say, "Try a little bit harder, and you can make it."

Teacher A said, "I say, 'Only a little more practice,' or 'You can do it the next time,' in order to motivate them." Teacher B said, "I tell the children, 'If you try, you can do it,' in order to motivate unwilling children."

To the question, "Don't you think you are not giving enough information concerning ways and skills of physical activity and sports to unwilling children?" they answered as follows:

Teacher A said, "I tell them what I think is enough. ...I feel some children are capable of being instructed, mainly on skill instructions, but there are others who are not capable of receiving such instructions."

Teacher B said, "There are things they (unwilling kids) simply do not understand and just cannot do."

Concerning scolding of unwilling children, Teacher A said, "Yes, I think I scold them frequently ... because I am always feeling I want unwilling children listen to me carefully. That's why I scold them ...especially when it is the physical activity they are not interested. If they don't listen to me, we cannot proceed with the study ...That's why."

Teacher B said, "If I do not make unwilling children listen, all the other children won't listen."

Both of them said they scold unwilling children often.

The above learning behaviors that belong to this category applied to 7 out of 8 unwilling children.

\section{Discussion}

The recognition that the factors that cause children's dislike of PE classes and/or sports are the level of difficulty of motor task, learner group, and teaching principles of the teacher is supported by the results of studies of Kobayashi (1970, pp.289-292), Sakumoto and Shinozaki (1979, pp.61-63), Jinzenji et al. (1993, pp.30-31) and Hatano and Nakamura (1981, pp.180182).

Unwilling children are not anxious to achieve new motor tasks [Portman, (1995, pp.448-449)]. However, as we know that the learning behaviors of unwilling children are affected by the level of difficulty of motor task, learner group, and teaching principles of the teacher, we could improve the class so as to affect the learning behaviors of unwilling children for the nurturing of desirable attitude.

In cases of low-skilled children, there are times when part of the blame should be on the learner group [Portman, (1995 pp.448-449); Griffin, (1985, p.106)]. We need to note the possibility that the learner group is depriving the unwilling children of the learning opportunities or the motor learning opportunities, and improve the relevant class accordingly.

Unwilling children were obeying instructions given by the learner group that is depriving them of the learning opportunity. This is because: firstly, between the unwilling children and the learner group, it is considered acceptable that low-skilled children are given instructions concerning the learning behaviors; secondly, it is assumable that unwilling children have less knowledge of physical activities and sports (basic rules; basic moves). These reason need further review.

Portman (1995, p.450) has already pointed out that teachers do not give skill feedback to low-skilled children. Teachers mostly think that the motor skill of unwilling children will not improve easily even with skill feedback [Jinzenji et al., (1993, p.36)]. This reasoning by teachers is causing them to put more emphasis upon remarks to motivate them to participate than to teach them to improve their skill. Teachers assume under the above teaching principles they could prevent the lowering of learning volition as well as decrease of the level of learning engagement behavior.

The unwilling children are rarely engaged in motor tasks that exceed their performance, and are deprived of motor learning opportunities by instructions made by the learner group, and are given little feedback for 
the improvement of the skill from teachers. This is the situation they are in, which has been created by the children's lower motor skills, which in turn is creating a cycle of increasing downgrading of their performance [Hatano and Nakamura, (1981, p.184); Portman, (1995, p.452)]

On the other hand, unwilling children, in classes that have relations with the improvement of their performance, such as in classes with motor tasks they can achieve, in classes with motor tasks they have achieved, and on occasions when they were given instructions from teachers personally, showed positive learning behaviors.

Thus, it is necessary to give a class that guarantee the improvement of motor skills of unwilling children in order to improve the learning environment they are in. Specification of motor tasks, subdividing them, securing of learning opportunities for unwilling children, providing sufficient information on how to do motor tasks and the way to choose the motor task, and increase of specific and skill feedback from teachers are some of the solutions for the improvement of the environment the unwilling children are in.

\section{Conclusion}

The purpose of this study is to identify the cause that affects the learning behaviors of children who do not enjoy PE classes.

As a result, three categories were identified, namely, (1) the level of difficulty of motor task (2) learner group, and (3) teaching principles of the teacher, as factors that affect the learning behaviors of unwilling children.

These are related to motor skills of children and worked against the enhancement of learning engagement behaviors as well as that of performance.

It is necessary to study in the future if the improvement of learning behaviors of children can be achieved and if their willing and favorable attitudes toward PE class can be upgraded through the improvement of the class from the above viewpoint.

\section{References:}

Bogdan, R. C. and Biklen, S. K. (1992). Qualitative research for education (2nd edition). Allyn and Bacon: Boston. pp.153-183.

Griffin, P. S. (1985). Boys' participation style in a middle school physical education team sports unit. JTPE 4:100-110.

Hatano, Y. and Nakamura, M. (1981). Case studies on the formation of unfavorable attitude against physical activities. Jap. J. Phys. Educ. 26(3): 177-187. (in Japanese).

Jinzenji, H., Iino, T., Ohtaguro, Y., and Yamamoto, E. (1993). A study of students in Miyazaki Prefecture who dislike sports and physical education: The current situation and causal factors. Memoirs of the faculty of education Miyazaki university, Art, health and physical education, home economics, and technics 74: 23-43. (in Japanese).

Kobayashi, A. (1970). What does cause unfavorable attitude against physical activities? : The social condition. Journal of health, physical education and recreation 20(5): 289-293. (in Japanese).
Ministry of Education, Science and Culture, Government of Japan (1999). The course of study for elementary school. Tokyo: Jiji Press. (in Japanese).

Nishi, J. and Kikkawa, M. (1980). The relation between the enjoyment of physical activities in the lessons of physical education and lifelong sport. Research journal of sport sociology 9: 41-62. (in Japanese).

Portman, P. A. (1995). Who is having fun in physical education classes? : Experiences of six-grade students in elementary and middle schools. JTPE 14: 445-453.

Sakumoto, M. and Shinozaki, T. (1979). A study of "Sports-Harter" in school physical education (I). Science of human life: Fukuoka women's university 12(1): 55-78. (in Japanese).

Takada, T., Okazawa, Y., Takahashi, T., and Kanegae, J. (1991). Development of a new instrument for the pupil evaluation of the instructional effectiveness in physical education class. 1989-1990 Grant-in-Aid for Scientific Research from Ministry of Education, Science and Culture, Government of Japan. Research representative: Takahashi, T. "The basic studies for improving physical education class". pp.172-182. (in Japanese).

Takahashi, T., Okazawa, Y., and Otomo, S. (1989). The effectiveness of observation system of academic learning time in physical education. Jap. J. Phys. Educ. 34(1): 31-43. (in Japanese).

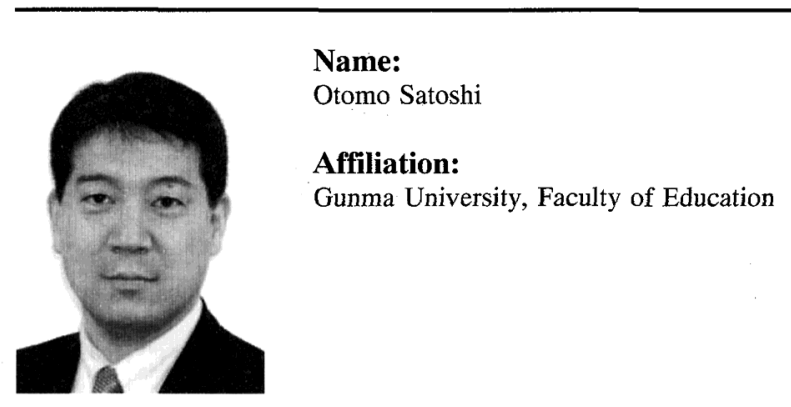

Address:

4-2, Aramaki-cho, Maebashi, Gunma, 371-8510 Japan

Brief Biographical History:

1989- Teacher at Todaiji Gakuen High School

1992- Naruto University of Education

1997- Visiting Scholar, The Ohio State University

1999- Gunma University, Faculty of Education

2002- Visiting Scholar, University of Tsukuba

Main Works:

- "Case studies of teacher behaviors in elementary school physical education classes", Japanese Journal of Sport Education Studies, Vol. 19, No.2, 159-173, (1999)

Membership in Learned Societies:

- Japan Society of Physical Education, Health and Sport Sciences

- Japanese Society of Sport Education(JSSE) 